\title{
Gravitational Model of the Three Elements Theory: Mathematical Explanations
}

\author{
Frederic Lassiaille \\ University of Nice Sophia Antipolis, Nice, France \\ Email: lumimi2003@hotmail.com
}

Received January 19, 2013; revised February 21, 2013; accepted March 19, 2013

Copyright (C) 2013 Frederic Lassiaille. This is an open access article distributed under the Creative Commons Attribution License, which permits unrestricted use, distribution, and reproduction in any medium, provided the original work is properly cited.

\begin{abstract}
The aim is to parse the mathematical details related to the gravitational model of the three elements theory [1]. This model is proven to be coherent and really compatible with relativity. The Riemannian representation of space-time which is used in this model is proven to be legal. It allows to understand relativity in a more human sensitive manner than Minkowskian usual representation.
\end{abstract}

Keywords: Relativity; Gravitation; Newton's Law

\section{Introduction}

The aim of this article is to depict the mathematical basis which supports the gravitational model of the three elements theory [1]. The first analysis addresses the link between the two space-time representations. Those representations are Minkowskian usual representation and a Riemannian representation. The latter must be detailed, and usual relativity mechanisms like general relativity principles and Lorentz transformation are interpreted in this representation in a particular geometrical manner. Lorentz transformation is explained by postulate 1 of [1] which is interpreted geometrically in a wired manner. This must be explained here. Also, application of postulate 3 of [1] uses a strange projection rule, which must be explained. The last analysis is checking that the "following geodesic principle" is still valid in this Riemannian representation, in a specific case. This is mandatory for the calculations which are done in [1].

\section{Comparison of the Two Metrics}

Let's call $g_{\lambda \mu}$ the Minkowskian pseudo-Riemannian metric coefficients, and $h_{\lambda \mu}$ the corresponding Riemannian metric ones.

As usual we have:

$$
\mathrm{d} s^{2}=g_{\lambda \mu} \mathrm{d} x^{\lambda} \mathrm{d} x^{\mu}
$$

With $x^{0}=c t$, and $x^{i}=x^{1}, x^{2}, x^{3}$ the three space coordinates. In this equation above $x^{\lambda}$ variables are supposed to be space and time coordinates in some given
$R_{0}$ reference frame. Let's choose a $B_{0}$ base as part of this $R_{0}$ reference frame. In other words, $B_{0}$ is the $R_{0}$ reference frame at some given instant in time.

In each space-time point, a mathematical theorem states that, for the bilinear form associated with this $\mathrm{ds} \mathrm{s}^{2}$ quadratic form, it is always possible to find an orthogonal and normalized $B$ base with respect to some fixed $B_{0}$ base, for which the metric local matrix is diagonal. In these orthogonal bases, the metric coefficients becomes then such that $g_{\lambda \mu}=0$ if $\lambda \neq \mu$. That's just a reminder. But let's notice that time and space units in these $B$ bases are $B_{0}$ base time and space units. In those bases, now the Minkowskian metric can be written:

$$
\mathrm{d} s^{2}=g_{00} c^{2} \mathrm{~d} t_{b}{ }^{2}+g_{11} \mathrm{~d} x_{b}^{12}+g_{22} \mathrm{~d} x_{b}^{22}+g_{33} \mathrm{~d} x_{b}^{32}
$$

$t_{b}, x_{b}^{1}, x_{b}^{2}, x_{b}^{3}$ are coordinates in the $B$ base. By definition, the Riemannian coefficients are constructed the following way.

$$
\begin{gathered}
h_{00}=\frac{1}{g_{00}}, \\
h_{i i}=-\frac{1}{g_{i i}}, i \neq 0
\end{gathered}
$$

Those equations are a consequence of the differences between Minkowskian representation and the Riemannian representation which is used in this model. The first one is of course the metric signature which is ++++ for the Riemannian one, in place of the +--- Minkowskian one. The second difference is that local time is inverted. In other words, the diagonal matrix coefficients 
are inverted when passing from one metric to another. This allows to apply the "following geodesics" principle in this Riemannian representation, as this will be shown in this document. The physics understanding of this second difference is the same as the general relativity explanation of the twin paradox. Let's remind that this explanation is based upon the generation of a global vacuum by the universe in the representation of the reference frame attached to the travelling twin. On the contrary the twin staying on earth is watching the vacuum generated by the travelling twin only locally and that's the explanation of the asymmetrical roles of the twins. This is not a physical vacuum but a vacuum in the representation of space-time. Now in the Riemannian representation, with respect to $R_{0}$ reference frame, this vacuum is supposed to be generated globally by the free falling particle (as if the free falling particle was not less than the universe itself!). On the contrary, in the Minkowskian representation in $R_{0}$ this vacuum is supposed to be generated only locally to the particle along its trajectory. Of course, only the latter version is the realistic one. But one may notice that the mathematical model do not prefer any physical configuration.

An illustration of this relationship rule between those two metrics is given by the case of the Schwarzschild metric.

$$
\mathrm{d} s^{2}=\left(1-\frac{M}{x}\right) c^{2} \mathrm{~d} t_{b}^{2}-\left(1-\frac{M}{x}\right)^{-1} \mathrm{~d} x_{b}^{2}
$$

Of course this is the Minkowskian metric usual version.

$M$ is supposed to be the Schwarzschild ray of the attracting object as usual. $x$ is as usual in this metric the spatial physical distance from the attracting object (more usually written $r$ ). $c t_{b}=x_{b}^{0}$, and $x_{b}=x_{b}^{1}$ are, respectively, the time and space coordinates in the system of $B$ bases. $d s$ is the infinitely small space-time length, calculated in some given space-time point. The $R_{0}$ reference frame which generated this system of $B$ bases gets on its origin its time axis parallel to local time axis in two cases. The first one is when the origin of $R_{0}$ is located in the middle of the attracting object of this Schwarzschild metric. The second one is when the origin of $R_{0}$ is located infinitely far from the attracting object. In the latter case, $R_{0}$ time axis is tangent to the trajectory of a null mass free falling particle. Because of the equivalence principle, this free falling particle trajectory will coincide with a time coordinate curve in the system of $B$ bases. Because of the "following geodesics" principle, it will also be a geodesic in the Minkowskian metric. As usual this free falling particle gets a null mass, because we don't want it to generate any space-time deformations around it. Otherwise, this should modify the studied metric. And it gets a null speed when located infinitely far, because this means that its trajectory will always be perpendicular to space lines with respect to $R_{0}$. In other words, this trajectory is a time curve in this $R_{0}$ representation. In this document, the expression "free falling particle" will always means in fact this particular case of free falling particle, and the expression "time line" will always means that specific kind of trajectory. The term "space line" will mean perpendicular curves to such time lines.

The corresponding Riemannian version of this Schwarzschild metric is the following.

$$
\mathrm{d} s^{\prime 2}=h_{00} c^{2} \mathrm{~d} t_{b}^{2}+h_{11} \mathrm{~d} x_{b}^{2}
$$

Here, $d s^{\prime}$ is also a function of space and time lengths with respect to the $B$ base. As it will be seen, $\mathrm{ds}^{\prime}$ allows to draw space and time lines in the space-time Riemannian representation, with respect to $R_{0}$. It is this drawing which will allow to measure space-time lengths in this representation.

Using Equations (3), (4), and (5) there is:

$$
\mathrm{ds}^{\prime 2}=\left(1-\frac{M}{x}\right)^{-1} c^{2} \mathrm{~d} t_{b}^{2}+\left(1-\frac{M}{x}\right) \mathrm{d} x_{b}^{2}
$$

$\theta$ and $\varphi$ spherical coordinates are not written here. Their coefficients will get opposite sign when passing from one metric to the other, because their Euclidean associated local coordinates have their Minkowskian coefficients equal to -1 . Therefore, they becomes equal to +1 during the metric transformation when applying Equations (3) and (4). Hence, the $\theta$ and $\varphi$ coefficients get also their sign modified but that's all.

\section{Distance notations}

- Affine distance generated by Euclidean canonical metric of $R_{0}: D($,$) .$

- Distance given by the Riemannian metric and Equation (7): $d($,$) .$

On Figure 1 are drawn qualitatively those space lines associated with this Schwarzschild Riemannian metric, with respect to $R_{0}$. It is only after this drawing, that space-time lengths can be measured by evaluating the distances in $R_{0}$ between space lines (for measuring time) and between time lines (for measuring space).

On Figure 2 are firstly added two space-time points, $A$, and $B$. $A$ and $B$ are supposed to be infinitely close one to each other, and located on the same time line. It can be checked on Figure 2 that the space-time length between $A$ and $B$ correspond roughly to the number of space lines passed through when going from $A$ to $B$. Therefore when space lines get close from each other, Riemannian local time is elapsing faster with respect to $R_{0}$. This is the case as we get $x$ weaker and weaker, that is, as we get closer to the attracting object. Of course, it goes reversely for space distances. That's for a qualitative understanding. 


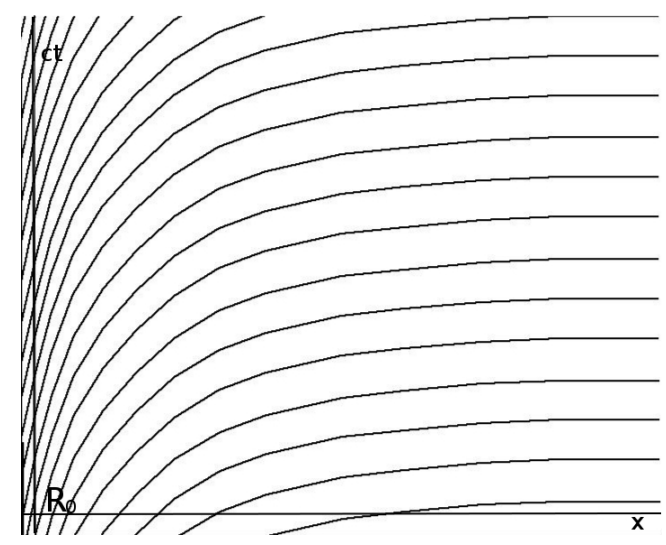

Figure 1. Space lines drawn with respect to $R_{0}$ reference frame.

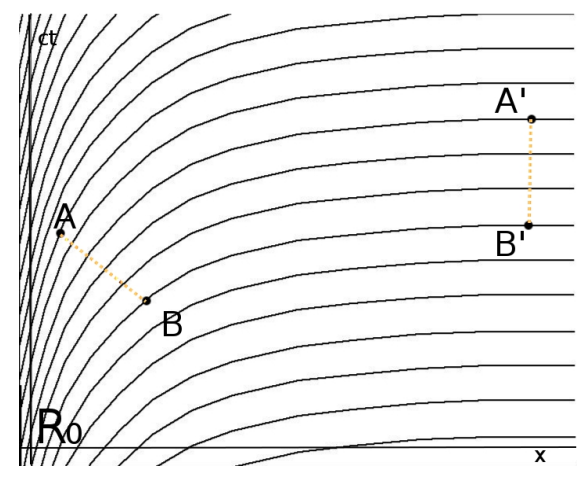

Figure 2. Two couples of points, each of them aligned on their local time line, $B$ and $B^{\prime}$ sharing the same space line, and such that $D(A, B)=D\left(A^{\prime}, B^{\prime}\right)$.

In a precise manner, the Riemannian metric distance between $A$ and $B$ is a $d s^{\prime}$ value calculated by Equation (7). Let's calculate this space-time length between $A$ and B:

$$
d(A, B)^{2}=\mathrm{d} s^{2}=\left(1-\frac{M}{x}\right)^{-1} c^{2} \mathrm{~d} t_{b}^{2}
$$

because here $\mathrm{d} x_{b}=0$, and then:

$$
d(A, B)=\frac{1}{\sqrt{1-\frac{M}{x}}} c \mathrm{~d} t_{b}
$$

Now let's redo this calculation for $A^{\prime}$ and $B^{\prime}$ points. Like $A$ and $B$, they share the same time line, but they are located far from the attracting object. It is supposed:

$$
D(A, B)=D\left(A^{\prime}, B^{\prime}\right)
$$

In other words, those segment distances are equal with respect to the Euclidean canonical metric given by $R_{0}$. If $x^{\prime}$ is the distance of the $B^{\prime}$ point from the attracting object, we get:

$$
d\left(A^{\prime}, B^{\prime}\right)=\frac{1}{\sqrt{1-\frac{M}{x^{\prime}}}} c \mathrm{~d} t_{b}^{\prime}
$$

Because that's Equation (9) applied to $A^{\prime}$ and $B^{\prime}$. Since $x^{\prime}$ is supposed to be far greater than $M$, then the following approximation can be done.

$$
d\left(A^{\prime}, B^{\prime}\right) \simeq c \mathrm{~d} t_{b}^{\prime}
$$

That's because here the space-time deformations generated by the attracting object are vanishing. It can be written by other means:

$$
c \mathrm{~d} t_{b}^{\prime} \simeq D\left(A^{\prime}, B^{\prime}\right)=c \mathrm{~d} t^{\prime}
$$

Indeed, here the $B$ base is the $B_{0}$ base except for their origins. Back to $B$ point, Equation (9) can be written the following way.

$$
\mathrm{d} \tau^{\prime}=\frac{1}{\sqrt{1-\frac{M}{x}}} \mathrm{~d} t
$$

This equation gives the time ratio between $\mathrm{d} \tau^{\prime}$, the local Riemannian time in $B$ point, and its corresponding $\mathrm{d} t$ time in $R_{0}$ reference frame. For yielding this equation, it has been used Equation (9),

$$
\begin{aligned}
& d(A, B)=c \mathrm{~d} \tau^{\prime}, \\
& c \mathrm{~d} t_{b}=D(A, B)=D\left(A^{\prime}, B^{\prime}\right)=c \mathrm{~d} t_{b}^{\prime}=c \mathrm{~d} t^{\prime}
\end{aligned} .
$$

$c \mathrm{~d} t^{\prime}$, time distance in $R_{0}$, has been renamed $c \mathrm{~d} t$ because this distance can be also evaluated in $\mathrm{B}$ point. Now let's calculate the local and physical time dilatation between $x$ and $x^{\prime}$ distances from the attracting object. The same reasoning with Minkowskian metric yields relativity time dilatation:

$$
\mathrm{d} \tau=\sqrt{1-\frac{M}{x}} \mathrm{~d} t
$$

Another way to understand the difference between Equation (14) and Equation (15) is the following. By construction, the physical local time ratio is growing inversely as compared with the Riemannian metric local time ratio. In other words, $d(A, B) / D(A, B)$ ratio must be inverted in order to get the $c \mathrm{~d} \tau / c \mathrm{~d} t$ ratio. That's because local times are inverted when passing from Minkowskian to Riemannian representation. In geometric words:

- The Riemannian time distance is growing proportionally with the number of crossed space lines;

- Local time physical length is growing proportionally with Euclidean distance between two given space lines.

Those values are inversely proportional to each other and Equation (15) could be retrieved this way starting from Equation (14). In other words, the relativistic coef- 
ficient $1 / \gamma$ is equal to $\mathrm{d} \tau / \mathrm{d} t$, as seen projected along local tangent of space lines. It can be said that it is also projected along space lines. This rule is illustrated by Figure 3.

$c \mathrm{~d} \tau$ represents, everywhere, the local physical distance between a given couple of space lines. This distance is measured with $R_{0}$ Euclidean metric. When those lines get close to each other, this value decrease because it is measured with $R_{0}$.

Inversely, the Riemannian distance, $\mathrm{d} s^{\prime}$, between those space lines remains the same everywhere. This distance is calculated with Equation (7) in a covariant manner along space lines.

\section{Covariance and Bases}

This covariance will be used for understanding this geometrical Riemannian mechanism. For this let's go back to Figure 2.

Let's write $B_{m}$ the base which is constructing, in $B$ point, the $R$ inertial reference frame of the free falling particle.

By construction, if $\boldsymbol{u}^{\lambda}$ are $B$ base vectors, and if $\boldsymbol{u}_{m}^{\lambda}$ are $B_{m}$ base vectors, there is $\boldsymbol{u}^{\lambda}=\sqrt{g_{\lambda \lambda}} \boldsymbol{u}_{m}^{\lambda}$. In other words, this $B_{m}$ base is orthogonal with its vectors normalized in the Minkowskian metric. This is not a new concept. It comes from general relativity in Minkowskian representation [2].

Now the same way, let's write $B_{r}$ the following base, in $B$ point. If $\boldsymbol{u}_{r}^{\lambda}$ are the $B_{r}$ base vectors, there is $\boldsymbol{u}^{\lambda}=\sqrt{h_{\lambda \lambda}} \boldsymbol{u}_{r}^{\lambda}$. In other words, this $B_{r}$ base is orthogonal with its vectors normalized in the Riemannian metric. $\mathrm{d} s^{\prime}$ distance between two space lines is the Riemannian length of $\boldsymbol{u}_{r}^{0}$ vector. By construction this length stays constant along a given space line.

Inversely, $c \mathrm{~d} t$ represents the distance between space lines calculated along $R_{0}$ time axis (not along local time axis). The $c \mathrm{~d} \tau$ vector is parallel transported along a

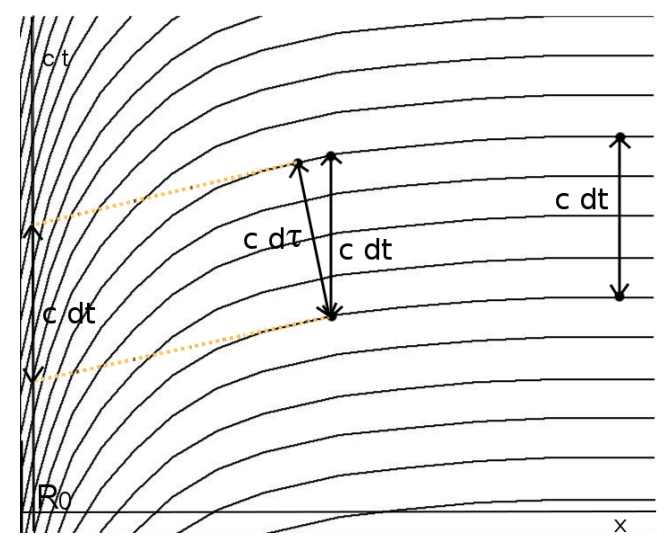

Figure 3. Local time, and global time. “ $c d \tau$ ” vector is parallel transported along space lines. given space line. The $c \mathrm{~d} t$ vector is the projected vector of this $c \mathrm{~d} \tau$ vector. This projection is done along the local space line tangents. But evaluation of this $c \mathrm{~d} t$ vector projection can be done everywhere along the corresponding space lines, in a parallel transport manner. This is also illustrated by Figure 3.

What about the space length ratio? The rule for the answer is the famous "constancy of metric determinant", which is a classical rule of general relativity. Using it, of course the consequence is merely an inverted evolution of the space length ratio as compared to the time ratio.

As an intermediate conclusion, the Riemannian distance allows drawing space lines. When drawing those lines, it is supposed that this Riemannian metric distance, $\mathrm{ds}^{\prime}$, between two given space lines is always the same when measured everywhere along those lines. Those space and time lines are drawn in the $R_{0}$ reference frame. This representation is understandable with human senses. In [1], it allows to understand the relativistic energy equation in the simple geometric manner of the Pythagore theorem. Let's remind that it is possible to describe this Pythagore equation, using surfaces, as a function of luminous points space-time deformation heights. This gives the determination of space-time shape in [1].

\section{How to Apply the Rules of the Postulate 3 [1]}

When applying the third postulate [1], the projection used for writing $\mathrm{d} x / \mathrm{d} s=1 / \gamma=\operatorname{oper}\left(L_{1}, L_{2}\right)$ was done along $R_{0}$ time axis, not along time lines.

On Figure 4 this postulate 3 application mode is shown.

That's because the determinant of the metric is supposed to be constant, as it was discussed above. Indeed, a geometrical property of this determinant is that it is the surface of the $A B C D$ rectangle of Figure 5 which is described by the $B_{r}$ base (of course this surface is measured in $R_{0}$ ).

But this surface is also the surface of the BDEF parallelogram of Figure 6 which vertical sides are given by the $c \mathrm{~d} t$ vector. The other vector side of this parallelo-

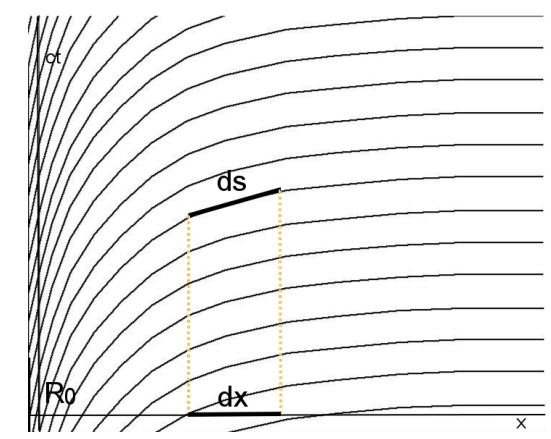

Figure 4. Application of Postulate 3 of [1]. 


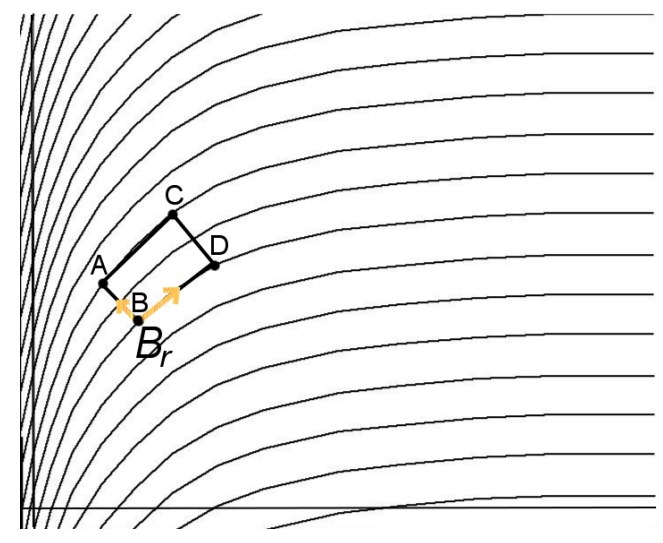

Figure 5. Geometric interpretation of the metric determinant: surface of the $A B C D$ rectangle drawn in the $B_{r}$ base.

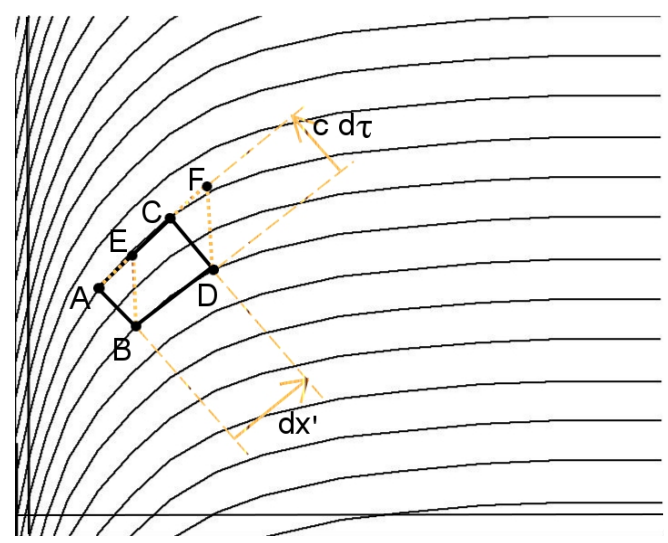

Figure 6. $B D E F$ parallelogram sharing the same surface as the $A B C D$ rectangle.

gram is $\mathrm{d} x^{\prime}$ which direction is along local space tangent. The projection of this vector along $R_{0}$ time axis is some $\mathrm{d} x$ fixed vector. This $\mathrm{d} x$ vector is parallel to $R_{0}$ space axis and its length stays constant through space and time. This is represented on Figures 5-7. Let's describe this more deeply.

On Figure 5, the surface of the $A B C D$ rectangle is equal to $D(A, B) D(B, D)=c \mathrm{~d} t \mathrm{~d} x^{\prime}$, the metric determinant.

$D(A, B)=c \mathrm{~d} t$ and $D(B, D)=\mathrm{d} x^{\prime}$ lengths are constructed from $B_{r}$ base vectors, and therefore from $h_{\lambda \mu}$ coefficients. There is: $D(A, B)=\left\|\boldsymbol{u}_{r}^{0}\right\|$, and $D(B, D)=\left\|\boldsymbol{u}_{r}^{1}\right\|$, with $\|\cdot\|$ being $D\left(\right.$,) (therefore $R_{0}$ ) associated norm. On Figure 6, the surface of the $A B C D$ rectangle is also equal to the surface of the $B D E F$ parallelogram.

On Figure 7, $c \mathrm{~d} t$ and $\mathrm{d} x$ remain constant when passing from $B D E F$ (parallelogram on the left) to $B^{\prime} D^{\prime} E^{\prime} F^{\prime}$ parallelogram (on the right). Inversely, $c \mathrm{~d} \tau$ and $\mathrm{d} x^{\prime}$ are modified. The metric determinant stay constant, it is equal to $c \mathrm{~d} t \mathrm{~d} x^{\prime}=c \mathrm{~d} t \mathrm{~d} x$ everywhere. It is the surface of

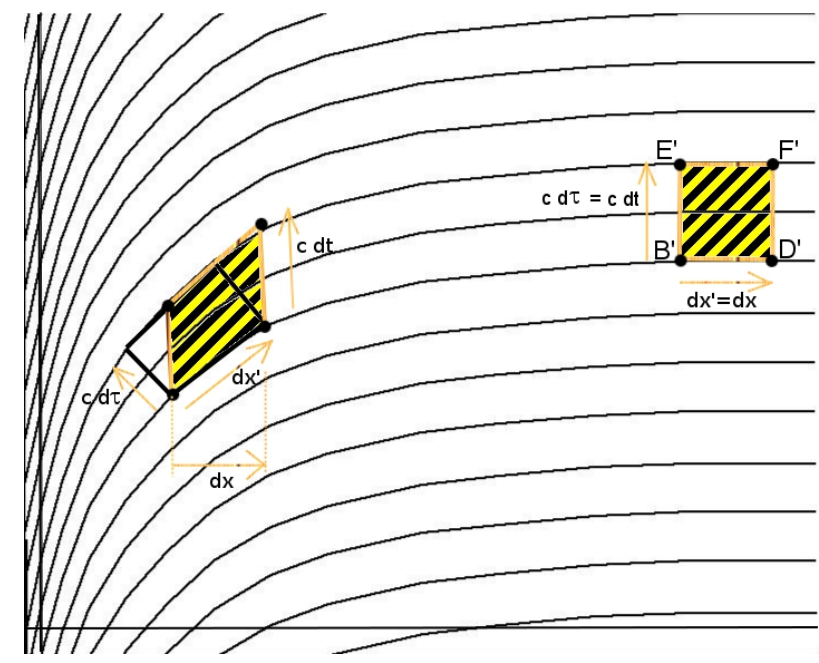

Figure 7. Interpreting constancy of metric determinant.

the $B D E F$ parallelogram, as well as the surface of the $B^{\prime} D^{\prime} E^{\prime} F^{\prime}$ parallelogram. In $B^{\prime}$, this $B^{\prime} D^{\prime} E^{\prime} F^{\prime}$ parallelogram is equal to the $A^{\prime} B^{\prime} C^{\prime} D^{\prime}$ rectangle (this $A^{\prime} B^{\prime} C^{\prime} D^{\prime}$ rectangle is not drawn).

The conclusion of this mechanism is the following.

- $R_{0}$ time lengths are projected along space lines, and

- Physical space lengths are projected along $R_{0}$ time axis.

In one word, this strange projection rule is explained by the constancy of metric determinant. Finally, if $v$ is the physical speed of the free falling particle with respect to $R_{0}$, and if $\alpha$ is the angle between local time axis and $R_{0}$ time axis, there is:

$\sin (\alpha)=\frac{\mathrm{d} s}{c \mathrm{~d} t}=\frac{\mathrm{d} x^{\prime}}{c \mathrm{~d} t}=\frac{v \mathrm{~d} t}{c \mathrm{~d} t}=\frac{v}{c}$, and not $\tan (\alpha)=\frac{v}{c}$. Here another legal used notation for $\mathrm{d} x^{\prime}$ is $\mathrm{d} s$, the infinitely small physical space distance along its space line with respect to $R_{0}$ reference frame. Therefore, the relativistic coefficient, yielded by the relativistic operator in [1], is equal as expected to $\frac{\mathrm{d} x}{\mathrm{~d} s}=\cos (\alpha)=\sqrt{1-\frac{v^{2}}{c^{2}}}$, and therefore there is $\sqrt{g_{00}}=\frac{1}{\sqrt{h_{00}}}=\sqrt{1-\frac{v^{2}}{c^{2}}}$.

\section{Comparison of Lorentz Transformation Formulation in the Two Metrics}

Let's go back to the geometrical interpretation of postulate 1 which is done in [1] in the context of the Riemannian metric.

Locally, the situation is exactly the same as above because of the postulate 1 of [1]. Indeed, in the context of Lorentz transformation, space-time deformation is locally exactly the same as studied above. Figure 8 shows qualitatively those space-time deformations. It could 


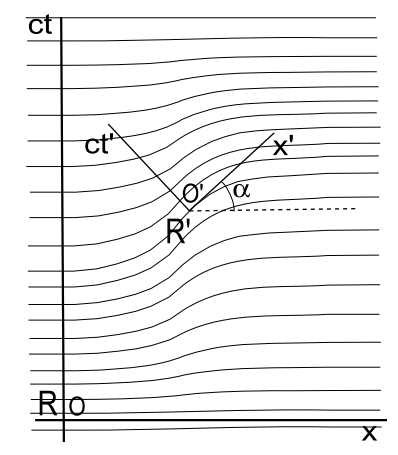

Figure 8. Space-time deformations in the case of Lorentz transformation, with respect to $R$ frame.

even be possible to write also metric equations for this case. Space line has rocked with an $\alpha$ angle such as $\sin (\alpha)=v / c$. Therefore, the same construction of vectors, bases and frames $\left(R_{0}, R, B_{0}, B, B_{m}, B_{r}\right.$, etc $\left.\cdots\right)$ still applies.

The usual $R(O ; c t, x, y, z)$ and $R^{\prime}\left(O^{\prime} ; c t^{\prime}, x^{\prime}, y^{\prime}, z^{\prime}\right)$ reference frames will be used as usual when writing Lorentz transformation:

$$
\begin{aligned}
& x^{\prime}=\frac{1}{\sqrt{1-v^{2} / c^{2}}}(x-v t) \\
& t^{\prime}=\frac{1}{\sqrt{1-v^{2} / c^{2}}}\left(t-\frac{v x}{c^{2}}\right)
\end{aligned}
$$

For the geometrical understanding of this transformation in [1], a projection of space-time lengths along the time and space axis of $R$ referential frame is used. But the normal usage of a basis should dictate to project those lengths along the time and space axis of $R^{\prime}$ referential frame.

And in the Riemannian representation which has been seen above, those projections were even more different. They were done, for $R$ time lengths along space lines (therefore along $R^{\prime}$ space axis), and for physical space lengths along $R$ time axis. Indeed, $R$ corresponds to $R_{0}$ in the description above, and $R^{\prime}$ corresponds to $B^{\prime}$ (which is just a reduced version of $B$ ).

Let's write Lorentz transformation in the context of the Riemannian metric. Using the strange projection rule of this representation, the space-time deformation of Figure 8, which is the postulate 1 of [1] space-time deformation, yields the following equations.

$$
\begin{aligned}
& x_{b}=\frac{1}{\sqrt{1-v^{2} / c^{2}}}(x-v t) \\
& t_{b}=\sqrt{1-v^{2} / c^{2}}\left(t-\frac{v x}{c^{2}}\right)
\end{aligned}
$$

Equations (17) are the result when applying the strange projection rule seen above, to the Figure 1 of [1] (or Figure 8). Let's remind that the coordinates of the
$O^{\prime}$ point are $\left(x_{o^{\prime}}, t_{o^{\prime}}\right)=\left(v t, v x / c^{2}\right)$. Therefore the first equation is the projection of local space lengths along $R_{0}$ time axis (and along Lorentz $R$ frame time axis). The second equation is the projection of time lengths along space lines and along Lorentz $R^{\prime}$ frame space axis. Let's remind that the final $\left(x_{b}, t_{b}\right)$ variables of (17) are the coordinates of space-time events in the $B$ base. Here, space lines where parallel to $R_{0}$ space axis, before the deformation, which is only local to the $O^{\prime}$ point. Therefore the speed vector of the $O^{\prime}$ point is parallel to $R_{0}$ space axis. This remark allows to write Equations (17). Now let's write $\left(x_{r}, t_{r}\right)$ the $B_{r}$ base coordinates. This transformation becomes the following.

$$
\begin{aligned}
& x_{r}=x-v t \\
& t_{r}=t-\frac{v x}{c^{2}}
\end{aligned}
$$

These equations are obtained using $x_{r}^{\lambda}=\sqrt{h_{\lambda \lambda}} x_{b}^{\lambda}$ and Equation (17). This is a Galilean transformation, which corresponds to the motion of the $O^{\prime}$ point which is the local point of the $R^{\prime}$ reference frame. But this motion is interpreted now with covariant space and time units. Indeed, those $\left(x_{r}, t_{r}\right)$ coordinates are those of the $B_{r}$ base which is part of a space-time map. By construction, in this map the resulting metric is the Euclidean trivial tensor, constant everywhere in space-time. It is therefore coherent to find here a Galilean transformation. This last formulation of Lorentz transformation is the corresponding one in this Riemannian representation of space-time.

Now (16) is derived from (18). First of all, (18) is similar to the affine transformation which is the identity linear application composed with the $O$ to $O^{\prime}$ translation. But it is noticed that the $O^{\prime}$ point is moving and its coordinates are function of $x$ and $t$. Therefore, (18) is in fact a linear transformation with respect to the $x$ and $t$ variables. As such, its determinant is no longer 1, which was the identity linear transformation determinant. Now its determinant is equal to $1-v^{2} / c^{2}$. Therefore, composition of this transformation (18) with $1 / \sqrt{1-v^{2} / c^{2}}$ which multiplies the Identity transformation yields (16), which is the same as (18) but with a determinant equal to 1 . And of course this final (16) result is Lorentz transformation.

This correspondence between those different formulations of Lorentz transformation gives the explanation of the strange projection rule: the geometrical Euclidean interpretation is driven by Equation (18). But this equation is another formulation of Lorentz transform.

\section{Comparison of the Geodesics between the Two Metrics}

Now let's compare the "following geodesics" principle in those two metrics. This comparison is mandatory be- 
cause this principle is used with the Riemannian metric when writing Equation (15) of [1].

The simplest way to express the "following geodesics" principle is the following. The free falling particle trajectory is a geodesic in the Riemannian metric. That's the most natural and simple way to express the "following geodesics" principle.

Of course, this is not the official one. The official one is the following. The free falling particle trajectory is an extremal trajectory in the Minkowskian metric. Now, the usual following reasoning must be done. It is always possible to construct a null trajectory close to any other trajectory. Since the free falling particle trajectory is a strictly positive one, and because of the signature +--- , (which is not -+++ otherwise the trajectory would become a minimal one), therefore its extremal value cannot be a minimal one. Therefore this is a maximal one. This was a reminder. This complicated and mathematical reasoning will be compared further with the one given by the gravitational model of the three elements theory.

Now it must be checked that those two geodesic definitions coincide. That is to say:

1) maximal trajectory in the Minkowskian metric,

2) minimal trajectory in the Riemannian metric, are exactly the same.

This is false in the general case. But it is true for a time line and in the case of the weak space-time deformations.

For proving this let's compare the geodesic trajectories in the two metrics. Let's remind usual equations of extremal trajectories in some given metric:

$$
\frac{\partial^{2} x^{\lambda}}{\partial \tau^{2}}=-\Gamma_{\mu \nu}^{\lambda} \frac{\partial x^{\mu}}{\partial \tau} \frac{\partial x^{v}}{\partial \tau}
$$

$\lambda$ being 0 for $c t$, or $i$ for $x^{i}$ as usual, and $\Gamma_{\mu v}^{\lambda}$ being the Christoffel symbols. $\tau$ is the exponential map metric parameter. In the Minkowskian one, it is equal to the physical local time. In the Riemannian one, along a time line the $\tau^{\prime}$ local time is different, related to the first one with $\mathrm{d} \tau^{\prime}=h_{00} \mathrm{~d} \tau$. Let's remind that the difference between those local times is driven by the importance of the space-time vacuum generated along time lines. The extremal (maximal) trajectory in the Minkowskian metric is the following.

$$
\begin{aligned}
& \frac{\partial^{2} x^{0}}{\partial \tau^{2}}=-\frac{c^{2}}{2} g_{00}^{-2} \frac{\partial g_{00}}{\partial x^{0}} \\
& \frac{\partial^{2} x^{1}}{\partial \tau^{2}}=-\frac{c^{2}}{2} \frac{\partial g_{00}}{\partial x^{1}}
\end{aligned}
$$

It has been supposed $\partial g_{00} / \partial x^{i}=\partial g_{11} / \partial x^{i}=0$ for $i=$ 2 and $i=3$. Therefore, the corresponding equations for $i$ $=2$ and $i=3$ become trivial and are not written here. It has been supposed also that $\partial x^{1} / \partial \tau^{\prime}=\partial x^{1} / \partial \tau=0$ along the trajectory, meaning that this trajectory is a time line.
Under those considerations, (20) equations are coming from (19) equations after calculations. The details of these calculations are available in [3]. Now the extremal (minimal) trajectory in the Riemannian metric is the following (expressed with the Minkowskian coefficients and Minkowskian local time for comparison).

$$
\begin{aligned}
& \frac{\partial^{2} x^{0}}{\partial \tau^{2}}=-\frac{c^{2}}{2} g_{00}^{-2} \frac{\partial g_{00}}{\partial x^{0}} \\
& \frac{\partial^{2} x^{1}}{\partial \tau^{2}}=-\frac{c^{2}}{2} g_{00}^{-4} \frac{\partial g_{00}}{\partial x^{1}}
\end{aligned}
$$

It has been supposed also that the trajectory is a time line in the Riemannian metric. The difference between (20) and (21) trajectory equations is only occurring for their second equations and is summarized this way:

$$
\frac{\partial^{2} x_{m}}{\partial \tau^{2}}=g_{00}^{4} \frac{\partial^{2} x_{r}}{\partial \tau^{2}}
$$

where $x_{m}$ is the value of $x^{1}$ in the Minkowskian metric trajectory, and $x_{r}$ its value in the Riemannian one.

Therefore, it is impossible to detect this difference in the weak deformations case. For example, in the Schwarzschild metric, there is $g_{00}^{4}=\left(1-\frac{M}{x}\right)^{4} \simeq 1$ for involved physical distances. (Of course, $x$ is the distance from the attracting object, and $M$ is the Schwarzschild ray). In a more convincing manner, the (20) second equation becomes the following.

$$
\frac{\partial^{2} x}{\partial \tau^{2}}=-\frac{M c^{2}}{2 x^{2}}
$$

Which is Newton's acceleration. Whereas the (21) second equation becomes the following.

$$
\frac{\partial^{2} x}{\partial \tau^{2}}=-\frac{M c^{2}}{2 x^{2}}\left(1-\frac{M}{x}\right)^{-4} \simeq-\frac{M c^{2}}{2 x^{2}}
$$

Which is an approximation of Newton's acceleration. Therefore, those equations are approximately equal.

Finally, the second equation of (20) can be written using the angle of the space curve tangent in this spacetime Riemannian representation. The result is that Equation (15) of [1] is also a good approximation of the last equation of (20), in the weak space-time deformations case.

Nevertheless, Equations (20) and (21) do not yield exactly the same trajectories. Of course, the question of which system equation is correct is easy to answer. The Minkowskian metric trajectory is the correct one. This choice can be argued with the help of the gravitational model of the three elements theory. Indeed, in this model the classical space-time distance

$$
\mathrm{d} s=\sqrt{g_{00} c^{2} \mathrm{~d} t^{2}+g_{11} \mathrm{~d} x^{2}} \text { depends upon } c \mathrm{~d} \tau=\sqrt{g_{00}} c \mathrm{~d} t \text {, }
$$


related to the total energy of the particle, and $\sqrt{-g_{11}} \mathrm{~d} x$, the height of the asymmetrical space-time vacuum generated by the motion of this free falling particle. In this model, this vacuum has always a key importance.

Therefore it is very coherent to have it minimized in the trajectories. This straightforward physical reasoning must be compared with the mathematical and complicated official one, which was reminded above.

As a consequence the Minkowskian metric is still of extreme importance and can't be replaced by the Riemannian one.

\section{Conclusions}

The aim given in the introduction has been achieved in a coherent manner. This proves that the gravitational model of the three elements theory is coherent and therefore really compatible with relativity. The Riemannian representation of space-time which is used in this model is legal. It allows to understand relativity in a more human sensitive manner than Minkowskian usual representation. Postulate 1 of [1] geometrical interpretation and postulate 3 of [1] application rule has been explained. Equation (15) of [1] has been explained. It uses the "following ge- odesics" principle in the context of the Riemannian metric. And this has been proven to be a correct approximation for involved physical distances and for time line trajectories.

Moreover, this geometrical sensitive description of reality allows the construction of the three elements theory, a unifying theory [4]. This theory is fully understandable in this geometrical and deterministic manner. It gives a complete traceability of the mathematic models calculations along their physical explanations. It indicates that a more intimate link might exist between classical physics theories and reality.

\section{REFERENCES}

[1] F. Lassiaille, Journal of Modern Physics, Vol. 3, 2012, pp. 388-397. doi:10.4236/jmp.2012.35054

[2] S. Carroll, "Space Time and Geometry, an Introduction to General Relativity," Addison-Wesley, San Francisco, 2004.

[3] F. Lassiaille, "Gravitational Model of the Three Elements Theory: Mathematical Detailed Calculations," 2013. http://lumi.chez-alice.fr/anglais/MathDetailedCalc.pdf

[4] F. Lassiaille, “Three Elements Theory,” 1999. http://lumi.chez-alice.fr/3elt.pdf 


\section{Glossary}

$c$ : Speed of light.

$x^{\lambda}$ : Space-time coordinates.

$x^{i}$ : Space coordinates.

$t$ : Time variable with respect to $R_{0}$ reference frame.

$x$ : Space physical distance between a space-time point and the center of the attracting object in the Schwarzschild metric, with respect to $R_{0}$. It is calculated with the help of an integral along a given space line.

$R_{0}$ : Inertial reference frame "attached to" the universe. For example, in the case of the Schwarzschild metric, $R_{0}$ is also attached to the attracting object.

$B_{0}$ : Base located along $R_{0}$ trajectory. The set of those bases is the $R_{0}$ reference frame.

$R$ : Inertial reference frame of a "free falling particle" (see definition). This particle is supposed as usual as getting a null mass for avoiding modification of the spacetime structure. It is located at rest with respect to $R_{0}$ when located infinitely far.

$B$ : Orthogonal and normalized base with respect to $R_{0}$ Euclidean metric, in which the Minkowskian metric is diagonal.

$B_{m}$ : Normalized base with respect to the Minkowskian metric. The set of those bases along a given "time line" (see definition) will construct the $R$ reference frame. This base is constructed from $B$ base. Its vectors are parallel to $B$ vectors. Their lengths are inverted from $B$ vectors lengths, with respect to the Minkowskian metric. In other words, we get $\boldsymbol{u}^{\lambda}=\sqrt{g_{\lambda \lambda}} \boldsymbol{u}_{m}^{\lambda}$.

$B_{r}$ : Normalized base with respect to the Riemannian metric. In some way it explains time and space values in $R$ reference frame before space-time vacuum generation by the free falling particle. This base is constructed from $B$ base. Its vectors are parallel to $B$ vectors.
Their lengths are inverted from $B$ vectors lengths, with respect to the Riemannian metric. In other words, we get $\boldsymbol{u}^{\lambda}=\sqrt{h_{\lambda \lambda}} \boldsymbol{u}_{r}^{\lambda}$.

$B^{\prime}$ : The vectors of this base are equal to $\sqrt{1-v^{2} / c^{2}}$ which multiplies the vectors of $B$.

Free falling particle: in this document, this always means a special kind of free falling particle. Its trajectory coincides with a time coordinate curve in the system of $B$ bases. Because of the "following geodesics" principle, it is a geodesic in the Minkowskian metric. As usual this free falling particle gets a null mass, because we don't want it to generate any space-time deformations around it. Otherwise, this should modify the studied metric. And it gets a null speed when located infinitely far, because this means that its trajectory will always be perpendicular to space lines with respect to $R_{0}$. In other words, this trajectory is a time curve in this $R_{0}$ representation.

Space line: a space curve, which is the space three dimension manifold, represented by a curve after projection on the two dimension figures of this document. But in this document, this curve is always perpendicular to any local time axis in the $R_{0}$ representation.

Time line: the trajectory of a free falling particle (refer to the above definition of "free falling particle").

$\boldsymbol{u}^{\lambda}: B$ base vectors.

$\boldsymbol{u}_{m}^{\lambda}: B_{m}$ base vectors.

$\boldsymbol{u}_{r}^{\lambda}: B_{r}$ base vectors.

$g_{\lambda \mu}$ : Coefficients of the Minkowskian metric with respect to the $B$ base.

$h_{\lambda \mu}$ : Coefficients of the Riemannian metric with respect to $B$ base. 